\title{
Relation of the Characteristic Action of Biscoclaurine Alkaloids on the Erythrocyte Membrane and Their Incorporation into the Membrane
}

\author{
Takashi Sato, Yasunori Kanaho and Tatsuzo Fujii \\ Department of Biochemistry, Kyoto College of Pharmacy, Misasagi, Yamashina- \\ ku, Kyoto 607, Japan
}

\begin{abstract}
In vitro effects of biscoclaurine alkaloids on cell morphology and the osmotic resistance of human erythrocyte membranes were examined in relationship to their incorporation into the membrane.

All the alkaloids tested induced invaginational type of transformation and a change in resistance against hypotonic hemolysis as a function of the concentration, within a range of $10^{-5} \mathrm{M}$ to $2 \times 10^{-4} \mathrm{M}$, in the medium. Alkaloids also were incorporated dose-dependently into cells in the same concentration range; the major part of the incorporation was in the membrane fraction. The effects of the alkaloids on the membrane as wall es their affinity for the membrane, judged by the amount incorporated, decrease as follows: tetrandrine $>$ cepharanthine $>$ berbamine. Fangchinoline and isotetrandrine had an invaginating effect similar to that of cepharanthine. The shape-transforming effect of cepharanthine was antagonistic to that of snake venom phospholipase $A_{2}$ which induced crenation of the cells by hydrolyzing the outer layer phospholipids of the lipid bilayer of the membrane. This alkaloid did not inhibit the hydrolytic action of the enzyme on the membrane. These results indicate that the biological action of the alkaloids on the erythrocyte membrane may be due to their interaction with the lipid bilayer of the membrane.
\end{abstract}

Cepharanthine, a biscoclaurine alkaloid, is a known potent inhibitor of snake venom-induced hemolysis in vivo (7). The antitumor effect of many kinds of biscoclaurine alkaloids on Ehrlich ascites carcinoma and the correlation between their activity and chemical structure have been reported recently $(9,10)$. Utsumi et al. demonstrated that the antihemolytic action of cepharanthine is due to its membrane stabilizing activity, that cepharanthine inhibits the release of histamine from peritoneal mast cells of the rat and the release of $\mathrm{K}^{+}$from various cells (e.g. red blood cells, Ehrlich ascites tumor cells, AH-130 ascites hepatoma and liposome) induced by phospholipase $\mathrm{A}_{2}$ or lysolecithin $(13,14)$. They also suggested that cepharanthine decreases the fluidity of the biological membrane, which results in various induced membrane stabilizing action (13). We recently showed that biscoclaurine alkaloids induce an invagination type of shape change in human erythrocytes (5).

Based on the characteristic action of cepharanthine on biological membranes, we here report the influence of various biscoclaurine alkaloids (e.g. cepharanthine, berbamine and tetrandrine) on cell morphology, osmotic hemolysis, and the phospholipase $\mathrm{A}_{2}$-induced shape changes in human erythrocytes. 


\section{MATERIALS AND METHODS}

Erythrocytes. Erythrocytes obtained from freshly-drawn human blood were washed three times with $140 \mathrm{mM} \mathrm{NaCl}$ containing $10 \mathrm{mM}$ phosphate buffer, $\mathrm{pH} 7.4$ (PBS), then resuspended in PBS.

Ghosts. Hemoglobin-free ghosts were prepared by hemolyzing washed erythrocytes in

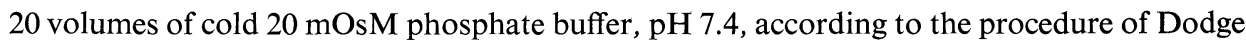
et al. (3). The ghosts obtained were resuspended in this buffer. Experiments on drug incorporation into the ghosts were performed in this hypotonic phosphate buffer.

Drugs. Biscoclaurine alkaloids were provided by Prof. Emer. Tomita and Kaken Pharmaceutical Co. Ltd. (Osaka, Japan). The alkaloids were dissolved in PBS for the experiments with erythrocytes, and in hypotonic buffer for experiments with the ghosts. The $\mathrm{pH}$ was adjusted to 7.4 with $\mathrm{HCl}$ or $\mathrm{NaOH}$. Phospholipase $\mathrm{A}_{2}$ (from Naja naja venom) was obtained from Sigma Chem. Co. Ltd. This was heated at $95^{\circ} \mathrm{C}$ for $30 \mathrm{~min}$ to inactivate contaminating proteases, after which it was dissolved in medium containing $0.25 \mathrm{mM} \mathrm{CaCl}_{2}, 0.25 \mathrm{mM}$ $\mathrm{MgCl}_{2}, 150 \mathrm{mM} \mathrm{NaCl}$ and $50 \mathrm{mM}$ Tris, $\mathrm{pH} 7.4$.

Osmotic fragility test. Various concentrations of alkaloids were dissolved in $10 \mathrm{mM}$ phosphate buffer, $\mathrm{pH} 7.4$, after which the tonicity of each solution was adjusted to $126 \mathrm{mOsM}$ with $\mathrm{NaCl}$. In the test solution of $126 \mathrm{mOsM}$, erythrocytes underwent about $50 \%$ hemolysis in the absence of alkaloids. A $0.2 \mathrm{ml}$ portion of $5 \%$ erythrocyte suspension was added to the hypotonic solution with or without alkaloid, then the mixture was incubated at $37^{\circ} \mathrm{C}$ for $5 \mathrm{~min}$. After incubation, erythrocytes were spun down at $900 \times \mathrm{g}$ for $5 \mathrm{~min}$, and the concentration of hemoglobin in the supernatant was determined by spectrophotometry at 543 $\mathrm{nm}$. The ratio of hemolysis in the presence of an alkaloid to that in its absence is the relative hemolysis.

Determination of alkaloids incorporated into erythrocytes or ghosts. Erythrocytes or ghosts in $10 \%$ suspensions were incubated with a drug for the period given in each experiment after which they were spun down at 900 or $15,000 \times \mathrm{g}$ for 10 or $30 \mathrm{~min}$, respectively. As the control, the drug solution in PBS or the hypotonic solution in the absence of erythrocytes or ghosts was used. The absorbances of the control and of the supernatant carefully removed from the alkaloid-treated erythrocyte suspension were measured by spectrophotometry at $282 \mathrm{~nm}$. The interfering ultraviolet absorbance of hemoglobin in the supernatant was determined by measuring the absorbance of the supernatant at $543 \mathrm{~nm}$ then calculating the value from it on the basis of the relationship between the absorbance of a known concentration of hemoglobin at $543 \mathrm{~nm}$ and at $282 \mathrm{~nm}$. Other interfering absorbances in the supernatant were determined by measuring the absorbance, at $282 \mathrm{~nm}$, of the supernatant of the $10 \%$ erythrocyte suspension in PBS, and of the $10 \%$ ghost suspension in the hypotonic solution, both incubated under similar conditions without alkaloids. The amount of the alkaloid incorporated was calculated from the difference between the absorbances of the control and the supernatant, obtained by subtracting the absorbance of hemoglobin and the other substances in the supernatant.

Morphological observation of erythrocytes. The shape of the erythrocytes was observed under a scanning electron microscope, type JSM-35, the Japan Electron Optic Lab. Co. (Tokyo, Japan), after fixation with $0.9 \%$ glutaraldehyde in $0.1 \mathrm{M}$ phosphate buffer, $\mathrm{pH} 7.4$, and a coating of carbon and gold. We used morphological indices for the semiquantitative representation of shape changes (5). The index was calculated from the stage of the transformed erythrocytes and from the percentage of total erythrocytes as follows: 
Morphological index

$$
=\sum\left(\text { Morphological score } \times \frac{\text { Number of transformed cells }}{\text { Total cell number }}\right)
$$

Crenation was scored from +1 to +4 , invagination from -1 to -4 , and the normal biconcave shape was scored 0 .

\section{RESULTS}

Effects of biscoclaurine alkaloids on the shape and osmotic hemolysis of human erythrocytes. Many amphipathic compounds with a cationic polar group induce an invagination type of shape change in human erythrocytes $(1,2,6)$. The alkaloids tested in this experiment are tertiary amines with two cationic polar groups in each molecule (Fig. 1). The morphological changes in erythrocytes after incubation with the alkaloids are shown in photographs in Fig. 2 and as morphological indices in Fig. 3. When normal biconcave erythrocytes (Fig. 2A and Index 0 in Fig. 3) were incubated with cepharanthine at $37^{\circ} \mathrm{C}$, they became cup-shaped (Fig. 2B and Index -1 in Fig. 3), then invaginated (Fig.2, C, D and Index -2 to -3 in Fig. 3), and finally smooth spheres (Fig. 2E) as the external concentration of this alkaloid increased. The shape-transforming potentials of isotetrandrine and fangchinoline were almost identical with the potential of cepharanthine. At the same concentration,

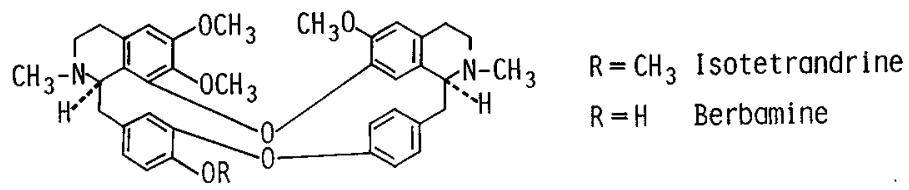

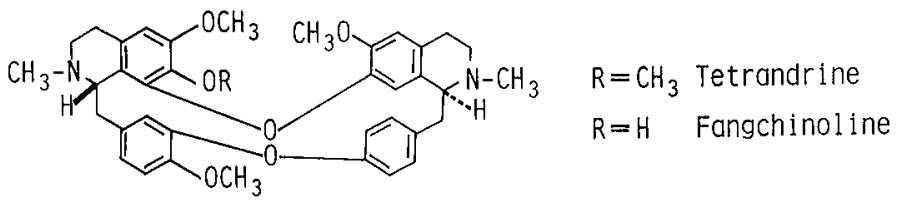

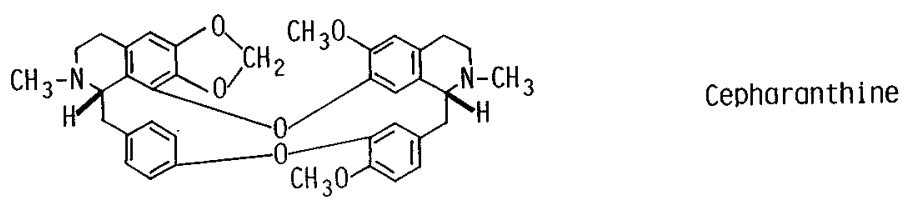

Fig. 1. Structures of the biscoclaurine alkaloids tested.
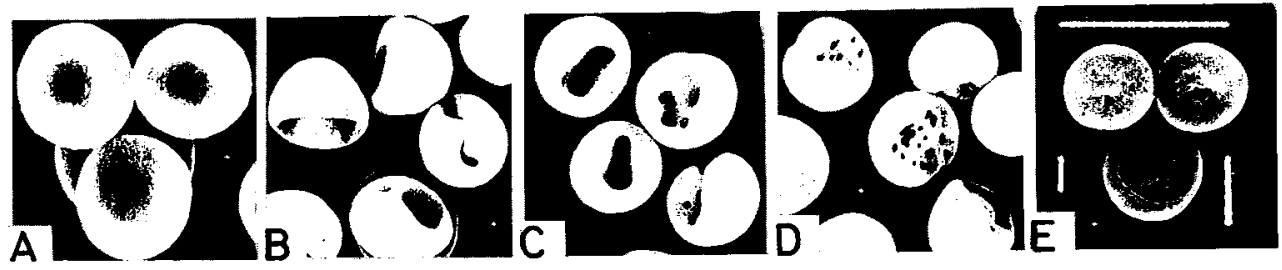

Fig. 2. Scanning electronmicrographs of human erythrocytes treated with cepharanthine. Normal erythrocytes (A) in a $10 \%$ suspension were incubated at $37^{\circ} \mathrm{C}$ for $10 \mathrm{~min}$ with $0.05 \mathrm{mM} \mathrm{(B),} 0.1 \mathrm{mM}$ (C), $0.2 \mathrm{mM}(\mathrm{D})$ and $0.5 \mathrm{mM}$ (E) of cepharanthine. 


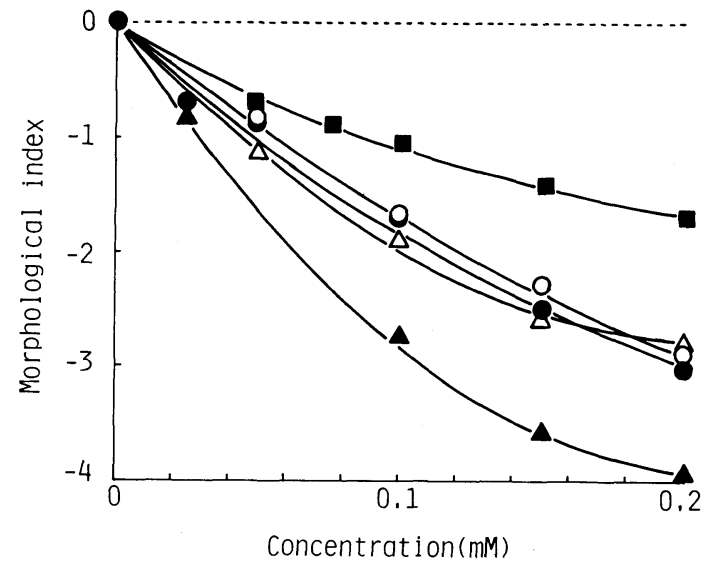

Fig. 3. Morphological changes in human erythrocytes induced by biscoclaurine alkaloids. Normal erythrocytes in a $10 \%$ suspension were incubated at $37^{\circ} \mathrm{C}$ for $10 \mathrm{~min}$ with berbamine $(\boldsymbol{\square})$, isotetrandrine $(\bigcirc)$, fangchinoline $(\triangle)$, cepharanthine $(\bullet)$ and tetrandrine $(\Delta)$. The resulting shape-change is a function of the external concentration of the alkaloid and is expressed by a morphological index.

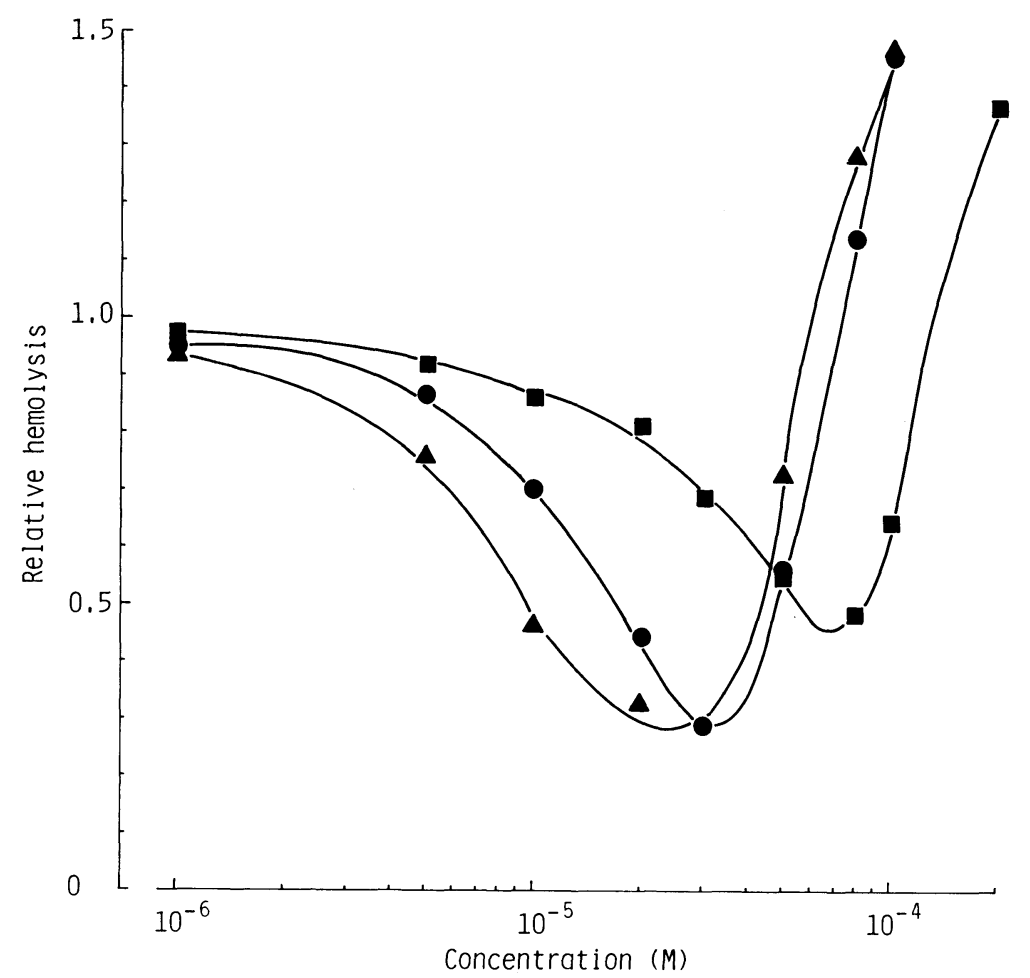

Fig. 4. The biphasic effect of biscoclaurine alkaloids on the hypotonic hemolysis of human erythrocytes. The percentage of hemolysis was determined in a hypotonic medium containing various concentrations of berbamine ( $\square$ ), cepharanthine $(\bullet)$ or tetrandrine (A). A hemolysis of about $50 \%$ in the hypotonic solution without alkaloid represents a relative hemolysis of 1. 
tetrandrine was more effective and berbamine was less effective than the other three. The shape changes took place too rapidly to be followed microscopically, and no further change in shape occurred during the $120 \mathrm{~min}$ of incubation.

The difference in the effects of these alkaloids on the erythrocyte membrane also was shown by the osmotic fragility test. All the alkaloids tested had two-phase effects on the osmotic behavior of erythrocytes; a protective effect at low concentrations and a stimulative effect at high concentrations (Fig. 4). Tetrandrine had the most intensive effect, berbamine was least effective for protection at low concentrations, whereas cepharanthine was more effective than berbamine but less than tetrandrine. The most effective concentration for protection was $2 \times 10^{-5} \mathrm{M}$ for tetrandrine, $3 \times 10^{-5} \mathrm{M}$ for cepharnathine, and $8 \times 10^{-5} \mathrm{M}$ for berbamine. The behavior of the alkaloids tested here was similar to that of other amphipathic drugs as reported elsewhere (11).

Incorporation of alkaloids into erythrocytes and ghosts. We examined whether the action of an alkaloid on cell shape and hypotonic hemolysis depends on the amount of the alkaloid incorporated into the erythrocytes or ghosts. When tetrandrine, cepharanthine and berbamine were incubated with intact erythrocytes or ghosts, each was incorporated dose-dependently (Fig. 5). Although the amount of each alkaloid incorporated into intact erythrocytes was almost the same (Fig. 5A), that incorporated into the ghosts decreased in the order of tetrandrine $>$ cepharanthine $>$ berbamine at the same concentrations (Fig. 5B). The amount of each alkaloid incorporated into the erythrocytes exceeded that incorporated into the ghosts in the high external concentrations; evidence that part of the alkaloid incorporated penetrated the intracellular fluid of the erythrocytes. Whether the alkaloid that penetrated the cytoplasma is bound to hemoglobin is not clear. The amounts of tetrandrine, cepharanthine and berbamine incorporated into ghosts corresponded to about 60,50 and $35 \%$, respectively, of the amount in the original external medium

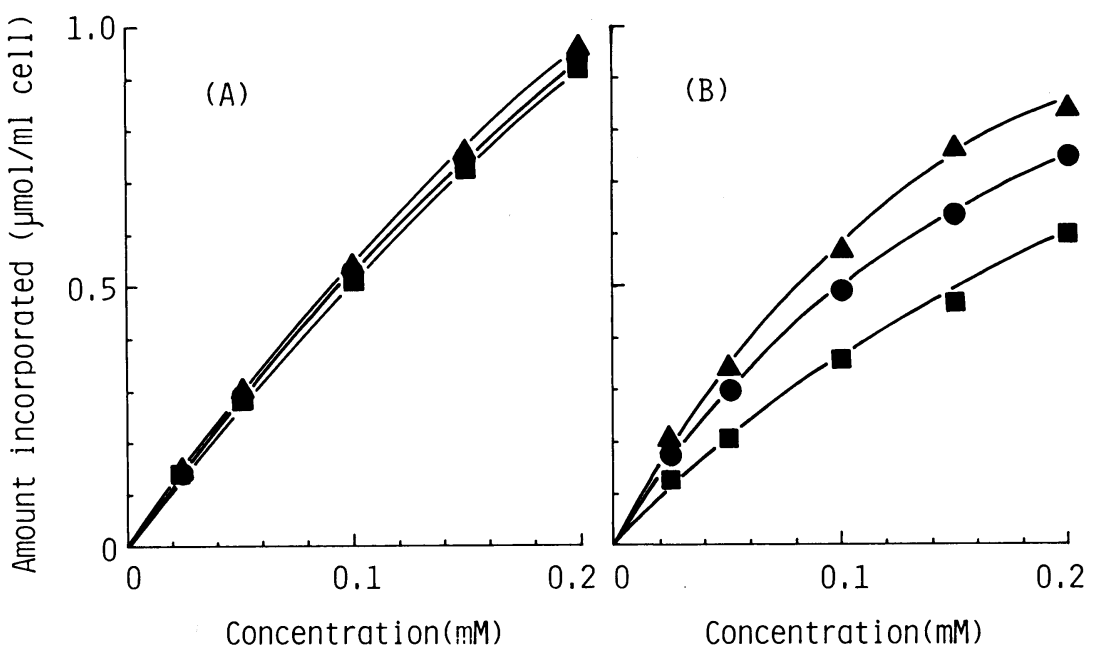

Fig. 5. Incorporation of biscoclaurine alkaloids into erythrocytes (A) and ghosts (B) as a function of the external concentration. Normal erythrocytes in a $10 \%$ suspension or ghosts equivalent to a $10 \%$ suspension of the original erythrocytes were incubated at $37^{\circ} \mathrm{C}$ for $10 \mathrm{~min}$ with tetrandrine $(\Delta)$, cepharanthine (๑) or berbamine (ם). After incubation, the erythrocytes and ghosts were centrifuged and the supernatant was used to determine the amount of alkaloid incorporated, as described in Materials_AND Methods. 


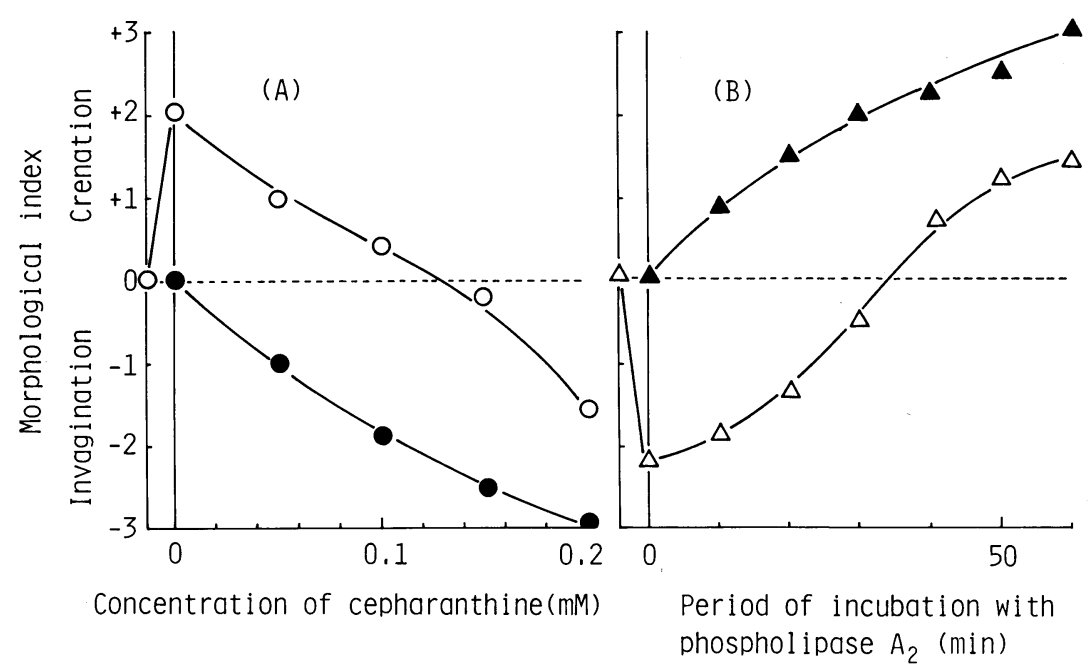

Fig. 6. Antagonism between the effects of cepharanthine and phospholipase $\mathrm{A}_{2}$ on the shape of human erythrocytes. (A) a $5 \%$ erythrocyte suspension in medium containing $5 \mathrm{mM} \mathrm{CaCl}, 150 \mathrm{mM}$

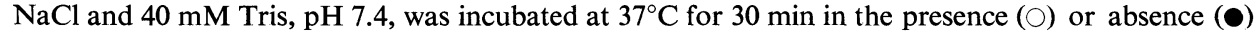
of phospholipase $\mathrm{A}_{2}$ (from Naja naja venom, $500 \mu \mathrm{g} / \mathrm{ml}$ cells), then it was washed three times with PBS to stop the enzyme reaction. The treated erythrocytes were suspended in $10 \% \mathrm{PBS}$ and incubated at $37^{\circ} \mathrm{C}$ for $5 \mathrm{~min}$ with various concentrations of cepharanthine. (B) the $10 \%$ erythrocyte suspension in the same medium as in (A) was incubated at $37^{\circ} \mathrm{C}$ for $5 \mathrm{~min}$ with $(\triangle)$ or without $(\Delta) 0.1 \mathrm{mM}$ cepharanthine. Phospholipase $\mathrm{A}_{2}$ then was added to the suspension to make a concentration of 500 $\mu \mathrm{g} / \mathrm{ml}$ cells and the mixture was incubated at $37^{\circ} \mathrm{C}$.

( $0.1 \mathrm{mM}$ concentration) under the conditions used here.

Effect of cepharanthine on phospholipase $A_{2}$-induced shape changes. One of us recently reported that the phospholipase $\mathrm{A}_{2}$ from snake venom caused erythrocytes to become echinocytic (4). Hence, a study with phospholipase $A_{2}$-induced transformed cells was carried out to investigate the possible antagonistic effect of cepharanthine against phospholipase $\mathrm{A}_{2}$ action. Incubation of erythrocytes with snake venom phospholipase $\mathrm{A}_{2}$ at $37^{\circ} \mathrm{C}$ for 30 min produced echinocytes, expressed by the morphological index +2 (Fig. 6A). When these echinocytes were incubated with cepharanthine at the concentration cited in the figure, they became a normal disc-shaped at $0.1 \mathrm{mM}$, and they were converted to cup-formed cells at $0.2 \mathrm{mM}$ (Fig. 6A). By contrast, the treatment of invaginated cells (of index -2), induced by cepharanthine $(0.1 \mathrm{mM})$, with phospholipase $\mathrm{A}_{2}$ at $37^{\circ} \mathrm{C}$ caused the cells to become echinocytic through an intermediate disc shape during the incubation period (Fig. 6B). In this case, hydrolysis of the membrane phospholipids of the cepharanthine treated cells at a given time proceeded to the same extent at in the untreated (control) cells.

Although similar antagonistic effects of tetrandrine and berbamine against phospholipase $\mathrm{A}_{2}$-induced shape change also were observed, the action of tetrandrine was more intensive than that of cepharanthine whereas berbamine was less (data not shown). 


\section{DISCUSSION}

We have presented evidence that cepharanthine and the other biscoclaurine alkaloids have biological action on erythrocyte membranes. We also confirmed that there is a close correlation between the action of the alkaloids on the membrane, such as the induction of morphological changes and protection against hypotonic hemolysis, and their incorporation into the membranes.

Positively-charged amphipathic compounds, mainly those with a cationized amine group in the molecule, are known to induce membrane invagination in human erythrocytes $(1,2,6)$. Since all the alkaloids tested in this study have two cationized tertiary amines in each molecule, morphological change is thought to be produced by these groups. The differences in the shape-transforming effect among the alkaloids may be due to their molecular structures. Since both berbamine and fangchinoline have a phenolic $-\mathrm{OH}$ group in their structures, they may be more hydrophilic than isotetrandrine and tetrandrine in which the same position is occupied by $-\mathrm{OCH}_{3}$. A difference in such a physical property should influence shape-transforming activity. A similar structure-activity relationship has been reported for erythrocyte membranes by Ben-Bassat et al. (1), who used pentaquine derivatives. Our results agree with theirs in so far as compounds with $-\mathrm{OCH}_{3}$ linked to benzene were more effective for invaginated shape changes of erythrocytes than those with -OH. However, our results do not show why, in spite of the similarity of their structures, tetrandrine has a more intensive effect on cell shape than isotetrandrine, and fangchinoline has a greater effect than berbamine. The structural difference between isotetrandrine and tetrandrine is at the absolute configuration of hydrogen at position 1 in the tetrahydroisoquinoline nucleus. The difference between berbamine and fangchinoline is at the position of the phenolic $-\mathrm{OH}$, in addition to the configurational difference. It is not clear how these structural differences produce differences in shape-transforming activities.

The activity of tetrandrine, cepharanthine and berbamine on the induction of the shape transformation paralleled their effects on protection against hypotonic hemolysis in a $10^{-5} \mathrm{M}$ to $2 \times 10^{-4} \mathrm{M}$ concentration range. Moreover, the biological activity of these three alkaloids on the membrane was related to the degree of their incorporation into ghosts in the same concentration range. If we assume that the amount incorporated into the ghosts is identical with that incorporated into the membrane of intact erythrocytes, then the biological actions of these alkaloids on the erythrocyte membrane must depend upon their affinities for the membrane.

Sheetz and Singer (12) have proposed that cationic amphipathic agents are incorporated into the cytoplasmic half of the lipid bilayer, expanding that layer relative to the other half, and thereby causing invagination of the membrane. According to this hypothesis, the alkaloids we tested probably would penetrate the cytoplasmic half of the lipid bilayer in the erythrocyte membrane. However, we have no direct evidence to indicate that the alkaloids incorporated into the ghosts are located in the inner leaflet of the lipid bilayer in the membrane.

Cepharanthine has been reported to have a therapeutic effect on snake venom injury and an inhibitory effect on snake venom-induced hemolysis (7). The anti-venom action of cepharanthine is believed to be caused by its inhibitory activity against the phospholipase A reaction on cell membranes. Our results, however, demonstrated that the cepharanthine incorporated into the erythrocyte membrane does not inhibit the enzymatic action of snake venom phospholipase $A_{2}$ itself, because the enzyme 
hydrolyzed membrane phospholipids of the cepharanthine-treated erythrocytes equally as well as it did those of intact erythrocytes. Thus, it is apparent that cepharanthine and phospholipase $A_{2}$ must act on the membrane independently, causing cells to change shape in opposite directions, consequently, they are antagonistic in their shape-transforming effects. If the phospholipase $A_{2}$-induced crenation is due only to structural alteration of the lipid bilayer, because of the hydrolysis of phospholipids in the outer leaflet of the bilayer, as claimed by Fujii and Tamura (4), then our results suggest that the crenated shape may return to normal, or further change to an invaginated shape by interaction of cepharanthine with the lipid bilayer of the membranes.

Possibly cepharanthine might interact with some of the proteins as well as with the lipids and cause invagination of the erythrocyte membrane. Since the membrane proteins are involved directly in the control of the passive $\mathrm{K}^{+}$permeability of erythrocytes, the structural changes in the membrane proteins probably induce an increased efflux of $\mathrm{K}^{+}$through the membrane. In fact, the sulf hydryl reactive reagent, $p$-chloromercuriphenylsulfonate, acts on erythrocytes and markedly increased the $\mathrm{K}^{+}$permeability of the membrane (8). In contrast, phospholipase $\mathrm{A}_{2}$-treatment of erythrocytes does not induce passive $\mathrm{K}^{+}$permeability change (unpublished data), which means that the significant efflux of $\mathrm{K}^{+}$is not induced only by phospholipid perturbation in the erythrocyte membrane. Therefore, the interaction of cepharanthine with some proteins in the erythrocyte membranes is unlikely when we consider that the alkaloid did not produce any significant efflux of intracellular $\mathrm{K}^{+}$during $2 \mathrm{~h}$ of incubation at $37^{\circ} \mathrm{C}$ at concentrations up to $0.2 \mathrm{mM}$ (unpublished data).

\section{REFERENCES}

1. Ben-Bassat, I., K. G. Bensch and S. L. Schrier. Drug induced erythrocyte membrane internalization. J. Clin. Invest. 51, 1833-1844, 1972

2. Deuticke, B. Transformation and restoration of biconcave shape of human erythrocytes induced by amphiphilic agents and changes of ionic environment. Biochim. Biophys. Acta 163, 494-500, 1968

3. Dodge, J. T., C. Mitchell and D. J. Hanahan. The preparation and chemical characteristics of hemoglobin-free ghosts of human erythrocytes. Arch. Biochem. Biophys. 100, 119-130, 1968

4. FujI, T. and A. TAmura. Asymmetric manipulation of the membrane lipid bilayer of intact human erythrocytes with phospholipase A, C, or D induces a change in cell shape. J. Biochem. 86, 1345-1352, 1979

5. Fuji, T., T. Sato, A. Tamura, M. Wakatsuki and Y. Kanaho. Shape changes of human erythrocytes induced by various amphipathic drugs acting on the membrane of the intact cells. Biochem. Pharmacol. 28, 613-620, 1979

6. Fuji, T., T. Sato and K. NaKanishi. In vitro shape changes of human erythrocyte membrane. Physiol. Chem. Physics 5, 423-430, 1973

7. HaSEgawa, S. and K. TAKAHASHI. The therapeutic application of cepharanthine for the snake venom injury. Saishin Igaku 7, 627-631, 1958

8. Knauf, P.A. and A. RothsteIn. Chemical modification of membranes. I. Effects of sulfhydryl and amino reactive reagents on anion and cation permeability of the human red blood cell. $J$. Gen. Physiol. 58, 190-210, 1971

9. Kupchan, S. M. and H. W. Atland. Structural requirements for tumor-inhibitory activity among benzylisoquinoline alkaloids and related synthetic compounds. J. Med. Chem. 16, 913-917, 1973

10. Kuroda, H., S. Nakazawa, K. Katagiri, O. Shiratori, M. Kozuka, K. Fujitani and M. 
TомітA. Antitumor effect of bisbenzylisoquinoline alkaloids. Chem. Pharm. Bull. 24, 2413-2420, 1976

11. Seeman, P. The membrane actions of anesthetics and tranquilizers. Pharmacol. Rev. 24, 583655,1972

12. Sheetz, M. P. and S. J. Singer. Biological membranes as bilayer couples. A molecular mechanism of drug-erythrocyte interactions. Proc. Nat. Acad. Sci. U. S. A. 71, 4457-4461, 1974

13. Utsumi, K., M. Miyahara, K. Sugiyama and J. Sasaki. Effect of biscoclaurine alkaloid on the cell membrane related to membrane fluidity. Acta Histochem. Cytochem. 9, 59-68, 1976

14. Utsumi, K., M. Miyahara, M. Inoue, M. Mori, K. Sugiyama and J. Sasaki. Inhibition by cepharanthine of red blood cell potassium release induced by lead acetate and lysolecithin. Cell Struct. Funct. 1, 133-136, 1975

(Received for publication, May 1, 1980) 